\title{
Efficacy of Surgical Revascularizations in Young and Middle-aged Patients with Symptomatic Middle Cerebral Arterial Occlusion
}

\author{
Mingze Wang \\ https://orcid.org/0000-0003-0086-8320 \\ Xingju Liu ( $\square$ liuxingju006@163.com ) \\ Yong Cao \\ Yan Zhang \\ Yuanli Zhao \\ Dong Zhang \\ Shuo Wang \\ Jizong Zhao
}

\section{Research}

Keywords: middle cerebral artery occlusion, young and middle-aged, revascularization, medication therapy, secondary ischemic stroke

Posted Date: June 14th, 2020

DOl: https://doi.org/10.21203/rs.3.rs-21536/v2

License: (a) (1) This work is licensed under a Creative Commons Attribution 4.0 International License. Read Full License 


\section{Abstract}

Background: Treatments on middle cerebral arterial occlusions (MCAO) remain controversial. This study aims at investigating efficacies of surgical revascularizations in young and middle-aged patients (aged 18-65 y) with symptomatic MCAO, and making comparisons between surgical procedures.

Methods: From a seven-year prospective consecutive registry, 115 cases were enrolled in this study. Patient individually received medical therapy alone (medical group, $n=64$ ) and medical therapy plus direct or indirect revascularization (surgical group, $n=51$ ). The secondary ischemic events (SIE) was defined as the primary outcome. SIE incidences and SIE-free survival rates were compared between groups. Risk factors were analyzed using the Cox regression analysis.

Results: During a follow-up of 42.7 months on average, SIE occurred in 11 cases of medical group and 10 cases of surgical group. SIE incidences were indifferent between groups (surgical:medical $=19.6 \%: 17 \%$, $\mathrm{P}=0.810,95 \% \mathrm{Cl} 0.455-3.034)$. SIE incidences were $29.4 \%$ and $0 \%$ in direct revascularization subgroup and indirect revascularization subgroup, respectively. Indirect revascularization induced a higher SIE-free survival rate than direct revascularization (Log-rank $P=0.015$ ) and medical therapy (Log-rank $P=0.044$ ). An age $>43 y$, with concomitant intracerebral arterial stenosis, and hyperlipidaemia history were correlated to the occurrence of SIE, while presences of compensatory arteries and taking antihypertensive drugs reduced risks of SIE.

Conclusions: Indirect revascularization plus medical therapy was suggested to be more effective on SIE prevention than both medical treatments alone and direct revascularization plus medical therapy in young and middle aged patients with symptomatic MCAO. Direct revascularization was not recommended, for its similar efficacy to medical therapy alone.

\section{Background}

Middle cerebral arterial occlusion (MCAO) induces ischemic stroke with an annual risk up to $12.5 \% .(1,2)$ For preventing the secondary ischemic events (SIEs), conventional medical treatments and surgical revascularizations have been applied with controversies on their efficacies. Even though case series have shown promising results of surgical revascularization for symptoms MCAO, (3-6) however, its benefits could not be proven by randomized clinical studies.(7-11) Aging-related factors are suspected to be responsible for the indifferent clinical outcome of surgical revascularizations, which would be less effects on MCAO patients younger than $65 \mathrm{y}$. The purpose of this study is to investigate the efficacy of surgical revascularizations on preventing SIE in young and middle-aged (aged 18-65y) symptomatic MCAO patients, and making comparisons between surgical procedures.

\section{Methods}

\section{Study design}


A single center prospective consecutive registry was reviewed. The registry included all patients diagnosed with symptomatic MCAO from January 2012 to December 2018 in Beijing Tiantan Hospital. Patients aged 18-65y were enrolled. Medical records, neuro-image, and results of the long-term follow-up were extracted for intergroup analyses. The study was approved by IRB of Beijing Tiantan Hospital. Written informed consents were obtained from all participants or their next-of-kin.

\section{Patient and public involvement}

Patients or members of the public did not participant in the design, or conduct, or reporting, or dissemination plans of the research.

\section{Study participants}

Patients enrolled in the registry cohort between Jan 2012 and Dec 2017 were further screened with inclusion and exclusion criteria. The inclusion criteria included: 1) aged 18-65y, 2) with MCAO diagnosed by computed tomographic angiography (CTA) or digital subtraction angiography (DSA), 3) with MCAOinduced ischemic symptoms or abnormal cerebral perfusions, and 4) received either surgical revascularization plus medical therapy or medical treatment alone. The ischemic symptom included transit ischemic attack (TIA) and ischemia-related neurological dysfunctions. The exclusion criteria included: 1) received endovascular treatment, 2) moyamoya disease or moyamoya syndrome was not excluded, or $3 \otimes$ with any history of arteritis, immune system diseases, or leptospira infection. Surgical revascularizations are recommended if the patients met any of these indications: 1) symptomatic MCAO, 2) with abnormal cerebral perfusion result, or 3 ) with aggravating symptoms which was uncontrollable with medical treatments alone. Preferences of patients were considered when making therapeutic decisions. The patients who refused operation would continue the treatment of medication. According to treatments performed, patients were divided into medical group and surgical group. In medical group, patients received medical therapy alone, including clopidogrel $75 \mathrm{mg}$, aspirin $100 \mathrm{mg}$ and atorvastatin $20 \mathrm{mg}$ every day. In surgical group, patients took aspirin $100 \mathrm{mg}+$ atorvastatin $20 \mathrm{mg}$ every day (except the operation day), and received either STA-MCA anastomosis (direct revascularization) or modified encephalo-duro-arterio-synangiosis (mEDAS, indirect revascularization). Conditions of STA and MCA, such as caliber and wall structure, determined the type of procedure. Indirect revascularization was performed when any of following conditions exists: 1) with insufficient caliber for anastomosis, 2) with abnormal vascular wall structure, 3 ) with compensatory collateral formation in operating field. To ensure the quality of direct revascularization, participating neurosurgeons must have performed $\geq 50$ anastomotic procedures in the latest 12 months.

Following information was extracted from the database. 1) Demographic information, including age, gender, race, and provenance. 2) Admission conditions, including primary symptoms, physical examinations and neurological function evaluations. Evaluations were conducted by neurological physicians using modified Rankin Scale (mRS). Neuroimaging results were independently reviewed by two sophisticated radiologists. 3) inhospital treatments, including medication and surgery. 4) Discharge conditions, including clinical outcomes, post-operative complications, physical examination and 
neurological function evaluations. Each lesion was documented as a single record for the patients with bilateral MCAOs.

\section{Follow-up}

Follow-up were conducted via two methods: 1) telephone follow-up: patients were contacted in every six months by full-time health visitors for self-assessments of mRS, and 2) outpatient follow-up: patients visited outpatient department for neurological and neuroimaging examinations, and neurological functional assessments via mRS in every six months. The outcomes were recorded in detail.

\section{Outcome evaluation}

The occurrence of SIE was the primary outcome event, defined as an aggravating recurrence or a new onset of both transient ischemic attack (TIA) and ischemic stroke, as well as a fatal final stroke, induced by ipsilateral MCAO. The secondary outcome event was set to be the SIE-free survival time, defined as the duration from receiving treatment to the occurrence of SIE or the end of follow-up; and

\section{Statistical methods}

All statistical analyses were performed using IBM® SPSS ${ }^{\circledR}$ Statistics (Version 22, IBM, NY, United States). The baselines of the two groups were compared using Student's t-tests and rank-sum tests. Pearson chi-square tests (or Fisher exact tests) were performed to compare the clinical outcomes between groups and subgroups. Kaplan-Meier estimates were performed to investigate differences in survival time. Receiver operating characteristic (ROC) analyses were used to obtain cutoff ages of different treatments. Cox regressions were performed to investigate correlated factors of SIE-free survival.

\section{Results}

124 patients were identified aged 18-65y. Nine patients were excluded for being treated with endovascular treatment, and 9 patients (7.8\%) were lost to follow-up in our study. Among 106 patients remained, nine patients were diagnosed with bilateral MCAOs. A total of 115 MCAOs (represented as Cases below) were involved (Fig. 1).

The 115 cases (male: female=76:39) enrolled were at a mean age of $47.5 \pm 9.9$ y (ranged 26-65y). Sixtyfour cases (male: female=41:23) were involved in medical group, with a mean age of $50.31 \pm 9.77 \mathrm{y}$. Fiftyone cases (male: female= 35:16) were involved in surgical group, with a mean age of $43.86 \pm 8.96$ y (Table 1). In the surgical group, 34 cases received direct revascularizations, and 17 cases received indirect revascularizations. 
Table1. Baseline Demographic of 115 Cases

Characteristic Medical Group Surgical Group Pvalue $(\mathrm{n}=64)^{\star} \quad(\mathrm{n}=51)^{*}$

Age, mean(SD), y.o.

$50.31(9.77)$

$43.86(8.96)$

0.001

Median (range), y.o.

$51.5(26-65)$

$45(26-59)$

Gender, male/female

$41 / 23$

$35 / 16$

0.506

Initial symptom

Stroke

TIA

Incident

Hypertension

Hyperlipidemia

Diabetes mellitus

Coronary artery disease

Unrelated cerebral infarction

Smoking history

Current

Former

Never

Drinking history

Never

Mild

Medium

Severe

Blood pressure, mean(SD), $\mathrm{mmHg}$

Systolic

Diastolic

BMI, mean (SD) ${ }^{\dagger}$

Median (range) ${ }^{\dagger}$
$35(54.7)$

$22(34.4)$

7 (10.9)

$44(68.8)$

30 (46.9)

$21(32.8)$

$3(4.7)$

$15(23.4)$

$16(25)$

15 (23.4)

$33(51.6)$

39 (60.9)

14 (21.9)

$8(12.5)$

$3(4.7)$

135.28 (16.08)

83.41 (11.73)

$26.2(4.3)$

$26.0(19.1-41.8)$

$8(15.7)$

0.161

4 (7.8)

0.021

39 (76.5)

0.005
0.060

0.205

0.149

0.595

0.001

0.432

0.119

0.001
1 (2)

0.005

43 (84.3)

0.014

3 (5.9)

0.097

2 (3.9)

0.547

3 (5.9)

Page $5 / 16$ 
Obese $(\geq 27.5 \mathrm{BMI})^{\dagger}$

NIHSS score, mean (SD) ${ }^{\ddagger}$

$1.05(2.00)$

$1.80(2.00)$

0.230

Range $^{\ddagger}$

0-13

$0-8$

mRS score, mean(SD)§

$0.69(0.77)$

$1.80(1.06)$

0.000

mRS score $\geq 2^{\S}$

$5(7.8)$

25 (49)

0.000

Compensatory collateral artery"

Intracranial"

Extracranial"

Concomitant cerebral arterial stenosis"

Ipsilateral"

Abnormal CTP result

Related cerebral infarction

Prehospital routine medication

\begin{tabular}{llll} 
Aspirin & $48(75)$ & $28(54.9)$ & 0.020 \\
\hline Clopidogrel & $33(51.6)$ & $3(5.9)$ & 0.000 \\
\hline Antihypertension & $26(40.6)$ & $19(37.3)$ & 0.431 \\
\hline Lipid-lowering & $51(79.7)$ & $18(35.3)$ & 0.000 \\
\hline Antidiabetes & $10(15.6)$ & $6(11.8)$ & 0.376 \\
\hline
\end{tabular}

Abbreviations: TIA, Transient ischemic attack; BMI, body mass index; NIHSS, National Institutes of Health Stroke Scale; mRS, modified Rankin's Scale; CTP, computed tomography perfusion.

${ }^{*}$ Data are reported as No.(\%) unless otherwise indicated.

${ }^{\dagger}$ BMI was calculated as weight in kilograms divided by height in meters squared. The threshold values of overweight and obese refer to National Standards published by National Health Commission of the People's Republic of China.

${ }^{\ddagger} \mathrm{NIHSS}$ score is based on a 42-point scale ( 0 indicates normal, and 42 indicates coma or death).

$\S \mathrm{mRS}$ score is based on a 7-point scale ( 0 indicates normal, $\geq 2$ is defined as neural function deficit, 6 indicates death). 
" Compensatory collateral artery is based on the images of digital subtraction angiography (DSA) or computed tomographic angiography (CTA).

'Related cerebral infarction is based on the results of computed tomography and magnetic resonance image.

During a mean follow-up of 42.7 months (ranged 2-80 months), the incidence of SIE was $17.2 \%(n=11)$ in medical group, and $19.6 \%(n=10)$ in surgical group (Table 2). The incidences of fatal stroke were $1.6 \%(1$ out of 64 ) and $3.9 \%$ (2 out of 51 ) in the medical and surgical group, respectively. SIE incidences were similar between groups (in total, $\mathrm{OR}=1.175,95 \% \mathrm{Cl} 0.455 \sim 3.034$; in SIE subtypes, $P=0.365$ ). In KaplanMeier estimates, there was no difference revealed between groups (Log Rank, $P=0.358$ and Breslow, $P=0.230$, Fig. 2a).

Table 2. Outcomes by Intergroup Analyses ${ }^{*}$

\section{Medical Group $(\mathrm{n}=64) \quad$ Surgical Group $(\mathrm{n}=51) \quad P$ Value}

\section{Primary outcome}

\begin{tabular}{|c|c|c|c|}
\hline 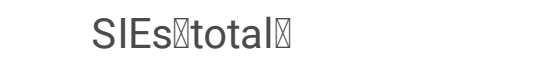 & 11 & 10 & 0.810 \\
\hline Fatal stroke ${ }^{\dagger}$ & 1 & 2 & 0.584 \\
\hline \multicolumn{4}{|l|}{ Secondary outcomes } \\
\hline Types of SIEs ${ }^{\ddagger}$ & 11 & 10 & 0.365 \\
\hline Aggressive recurrence & 7 & 9 & 0.420 \\
\hline New onset ${ }^{\dagger}$ & 4 & 1 & 0.392 \\
\hline $\begin{array}{l}\text { SIEs-free survival time } \\
\text { (months } \rrbracket\end{array}$ & $24.91 \pm 22.86$ & $43.50 \pm 25.98$ & 0.097 \\
\hline
\end{tabular}

Abbreviation: SIEs, secondary ischemic events.

* $P$ values were computed using Spears Chi-square test unless otherwise indicated.

† $P$ values were computed using Fisher's exact test

‡ Using 2’3 Chi-square tests

$\S P$ value was computed using Independent-sample t test 
Further subgroup analyses were performed in surgical group, between direct revascularization (DR) subgroup $(n=34)$ and indirect revascularization (IR) subgroup $(n=17)$. The baseline information of subgroups was different in age (DR group vs IR group $=41.91 \pm 9.44 \mathrm{y}$ vs $47.76 \pm 6.54 \mathrm{y}$, t-test, $P=0.026$ ) and hyperlipidaemia history (DR vs IR $=26.5 \%$ vs $0 \%$, Fisher's exact test, $P=0.017$ ). The incidences of $S I E$ were $29.4 \%$ and $0 \%$ in DR subgroup and IR subgroup, respectively (OR=0.706, 95\% $\mathrm{Cl} 0.568 \sim 0.877)$. Cases in IR subgroup had a higher cumulative SIE-free survival rate than those in DR subgroup (Log-rank $P=0.015$, Breslow $P=0.043$, Fig. $2 b)$. Compared with medical group, IR subgroup resulted in a lower incidence of SIE $(17.2 \%$ vs $0 \%, P=0.061,95 \% \mathrm{Cl} 0.741 \sim 0.926)$ and a higher SIE-free survival rate (Logrank $P=0.044$, Fig. 2C). Between medical group and DR subgroup, no significant difference was observed in either SIE incidence (17.2\% vs $29.4 \%, P=0.160,95 \% \mathrm{CI} 0.919 \sim 1.497)$ or SIE-free survival rate (Log-rank $P=0.097$, Breslow $P=0.065$, Fig. 2d).

ROC analyses revealed that cutoff ages were $48 \mathrm{y}$ and $43 \mathrm{y}$ in surgical group and medical group, respectively, which indicated a higher SIE incidence in the older cases. Among the cases aged 43 y -48 y, SIE incidences were indifferent between groups 『surgical groups vs medical group $=1 / 14$ vs 4/11, Fisher's exact test, $P=0.096 \rrbracket$. However, surgical revascularizations induced a higher SIE-free accumulative survival rate (Log Rank $P=0.067$, Breslow $P=0.033$ ). Further intersubgroup comparisons were conducted. The cutoff age of DR subgroup was identified to be $48 \mathrm{y}$, too. SIE incidences were 1/6 and 4/11 in DR subgroup and medical group, respectively, without significant difference (DR subgroup vs medical group $=1 / 6$ vs $4 / 11$, Fisher's exact test, $P=0.395,95 \% \mathrm{Cl}$ ). The SIE-free survival rates of DR subgroup and medical group were without significant difference, neither (Log Rank $P=0.379$, Breslow $P=0.230$ ).

Factors that affected patients' SIE-free survival were obtained in Cox regressions. The age $₫ 43 \mathrm{y}$ (HR=7.841, 95\% Cl 1.346-45.693), concomitant intracerebral arterial stenosis ( $\mathrm{HR}=5.358,95 \% \mathrm{Cl} 1.523-$ 18.858), and the history of hyperlipidaemia $(\mathrm{HR}=5.194,95 \% \mathrm{Cl} 1.394-19.358)$ were revealed to be risk factors of SIE-free survival. Meanwhile, taking antihypertensive drugs $(\mathrm{HR}=0.137,95 \% \mathrm{Cl} 0.038-0.489)$ and the existence of extracranial compensatory arteries $(\mathrm{HR}=0.216,95 \% \mathrm{Cl} 0.068-0.688)$ were revealed to contributors to survival.

\section{Discussion}

To our knowledge, this is the first study focusing on comparing efficacies of surgical revascularizations plus medical therapy versus medical treatments alone in young and middle aged patients with symptomatic MCAO. In our study, SIE occurred in $17.6 \%$ of patients received conventional medical treatment and $19.2 \%$ of patients received surgical revascularization plus medical therapy. The SIE incidences induced by medical and surgical treatments are in the ranges of $18.6-45 \%$ and $8.3-30.9 \%$, respectively, which are reported in published studies. $(1,5,6,8,9,12)$. In this study, the upper age boundary of inclusion criteria was lowered to $65 \mathrm{y}$ to investigate the efficacy of surgical revascularization in younger patients. It resulted in a younger mean age of participants than those in published studies. However, it failed to validate the superiority of surgical revascularizations in treating symptomatic MCAO 
patients aged 18-65 y. Underlying causes were analyzed via further interpretations of our data and inspections of published studies.

Although overall efficacies on SIE prevention were indifferent between groups in our study, a significant superiority was observed in indirect revascularization subgroup. The performing of indirect revascularization plus medical therapy resulted in a remarkably lower SIE incidence than both of direct revascularization subgroup and medical group. Indirect revascularizations is being complained about its incapability of improving distal hemodynamics immediately, $(7,13)$ so that it is commonly performed on the patients with poor intracranial and extracranial arterial condition who are unsuitable for STA-MCA anastomosis. In our experience, significant improvements of territorial perfusion have been observed in a lot of patients after mEDAS (a case example is presented in Fig.3). On one hand, indirect revascularizations gradually improves the territorial perfusion via the angiogenesis of pial arteries, instead of changing hemodynamics critically via anastomosis. $(14,15)$ On the other hand, the already formed compensatory arteries could be preserved in procedure, that improves the functional prognosis(16-18). The efficacy of indirect revascularization on treating MCAO needs investigating.

Direct revascularization has been proved to be effective on recovering the hemodynamics and preventing ischemic stroke. (19) However, only Japanese EC/IC Trial (JET) and JET-2 declare that direct revascularization results in more benefits than medical treatments on SIE prevention. $(5,6)$ Hemodynamic factors were emphasized in those studies, such as the threshold of cerebral vasodilatory reserve, cerebrovascular reactivity, cerebral blood flow, and oxygen extraction fraction. $(4,6,12,20)$ With strict hemodynamic criteria, direct revascularization could help preventing SIE.(21) In contrast, SIE occurred in $29.4 \%$ of patients receiving direct revascularization in our study. It is a higher incidence than that from Low et al. and JET-2, which is $13 \%$ and $8.3 \%$, respectively. $(6,12)$ Lacking of preoperative hemodynamic evaluation might be a main cause of the worse outcome. Hemodynamic indications of direct revascularization need to be explored.(11) Meanwhile, pathological characteristics of MCAO should be noticed as well. Latency occlusion of anastomotic window could not be avoided, though there are intraoperative indocyanine green angiograms and postoperative computed tomographic angiography performed. It is urged to develop indications with hemodynamic criteria and postoperative paradigms for long-term management to improve the outcome of direct revascularization.

Age-related effects should be considered when making the therapeutic decision of MCAO. The difference of medical and surgical treatment in their cutoff ages were revealed in our study, which developed into a 5 -year interval (surgical vs medical=48y vs 43y). The efficacy on SIE prevention decreased earlier in medical group than surgical group. It was confirmed by the higher SIE-free survival rate of patients aged 43-48y in surgical group. In the comparisons between DR subgroup and medical group, differences in SIE incidence and Kaplan-Meier estimate were observed. Although they were insignificant due to the small sample size of each group, it suggested additional benefits of direct revascularization might exist in a certain age interval. In the Cox regression, an age older than 43y was identified to be a risk factor for patients's SIE-free survival. It could be regarded as another evidence for the age-related effect on treating MCAO. Adaptable age phases of different treatments need to be further explored with larger sample size. 
Concomitant intracerebral arterial stenosis and hyperlipidaemia history were revealed to be related to incidents of SIE, which meet the results of published study.(22) Meanwhile, the protective factors deserved further discussions. Antihypertensive drugs have been reported to be effective on preventing SIE.(23) Among different classes, Tully et al.(24) reported that angiotensin receptor blocker (ARB) and beta-blocker were associated with ischemic stroke. Oppositely, Tziomalos et al.(25) reported that ARB was more effective on SIE preventing than any other classes. The class of antihypertensive drugs remains unclear. The existence of extracranial collateral compensatory arteries has been reported to play an unanimous beneficial role on preventing SIE.(18) It is proposed that the spontaneous formation of collateral arteries is not guaranteed, while indirect revascularization could help expediting the process. Further studies are needed.

Limitations exist in our study. 115 cases met the requirement of intergroup comparison, however, the sample size of each surgical subgroup was insufficient. Benefits of indirect revascularization were initially observed in our study, but it still need to be validated in further studies with larger sample size. Meanwhile, results might be limited since it is a single center registry. It is urgent to launch a multicenter randomized controlled trial for strong evidences.

\section{Conclusions}

Indirect revascularization plus medical therapy was suggested to be more effective on SIE prevention than medical treatments alone or direct revascularization plus medical therapy in young and middle aged patients with symptomatic MCAO. Direct revascularization was not recommended, for its similar efficacy to medical therapy alone.

\section{Declarations}

\section{Ethical approval and consent to participate}

The study was approved by IRB of Beijing Tiantan Hospital. Written informed consents were obtained from all participants or their next-of-kin.

\section{Consent for publication}

Not applicable.

\section{Data availability statement}

The data that support the findings of this study are available from the administrative department of Beijing Tiantan Hospital but restrictions apply to the availability of these data, which were used under license for the current study, and so are not publicly available. Data are however available from the 
authors upon reasonable request and with permission of the administrative department of Beijing Tiantan Hospital.

\section{Competing interests}

The work was not carried out in the presence of any personal, professional or financial relationships that could potentially be construed as a conflict of interest.

\section{Funding}

This work was supported by National Key Technology Research and Development Program of the Ministry of Science and Technology of China (2015BAl12B04), Beijing Municipal Organization Department talents project (2015000021469G219), Beijing Municipal ST Commission (D161100003816005), National Natural Science Foundation of China (81701137), Beijing Municipal Administration of Hospitals' Mission Plan (SML20150501), Beijing Institute for Brain Disorders (BIBDPXM2013_014226_07_000084).

\section{Author's Contribution}

MW participated in the study design, data acquisition and interpretation, and manuscript drafting. XL designed and conceptualized the study, interpreted the data and revised the manuscript. YC, YZ, DZ, YLZ and SW critically revised the manuscript for important intellectual content. JZ designed and conceptualized the study and critically revised the manuscript for important intellectual content.

\section{Acknowledgements}

This study was supported by the China National Clinical Research Center for Neurological Diseases. Dr. $\mathrm{HL}$ and $\mathrm{MZ}$ provided critical advices in the drafting of manuscript.

\section{References}

1. Kern R, Steinke W, Daffertshofer M, Prager R, Hennerici M. Stroke recurrences in patients with symptomatic vs asymptomatic middle cerebral artery disease. Neurology. 2005;65(6):859-64.

2. Weyer GW, Davis AM. Screening for asymptomatic carotid artery stenosis. JAMA. 2015;313(2):192-3.

3. Grubb RL, Jr., Powers WJ, Clarke WR, Videen TO, Adams HP, Jr., Derdeyn CP, et al. Surgical results of the Carotid Occlusion Surgery Study. J Neurosurg. 2013;118(1):25-33.

4. Kuroda S, Kawabori M, Hirata K, Shiga T, Kashiwazaki D, Houkin K, et al. Clinical significance of STAMCA double anastomosis for hemodynamic compromise in post-JET/COSS era. Acta Neurochir (Wien). 2014;156(1):77-83.

5. Ogasawara K, Ogawa A. [JET study (Japanese EC-IC Bypass Trial)]. Nihon Rinsho. 2006;64 Suppl 7:524-7. 
6. Kataoka H, Miyamoto S, Ogasawara K, lihara K, Takahashi JC, Nakagawara J, et al. Results of Prospective Cohort Study on Symptomatic Cerebrovascular Occlusive Disease Showing Mild Hemodynamic Compromise [Japanese Extracranial-Intracranial Bypass Trial (JET)-2 Study]. Neurol Med Chir (Tokyo). 2015;55(6):460-8.

7. Reynolds MR, Derdeyn CP, Grubb RL, Jr., Powers WJ, Zipfel GJ. Extracranial-intracranial bypass for ischemic cerebrovascular disease: what have we learned from the Carotid Occlusion Surgery Study? Neurosurg Focus. 2014;36(1):E9.

8. Group EIBS. Failure of extracranial-intracranial arterial bypass to reduce the risk of ischemic stroke. Results of an international randomized trial. N Engl J Med. 1985;313(19):1191-200.

9. Powers WJ, Clarke WR, Grubb RL, Jr., Videen TO, Adams HP, Jr., Derdeyn CP, et al. Extracranialintracranial bypass surgery for stroke prevention in hemodynamic cerebral ischemia: the Carotid Occlusion Surgery Study randomized trial. JAMA. 2011;306(18):1983-92.

10. Amin-Hanjani S, Barker FG, 2nd, Charbel FT, Connolly ES, Jr., Morcos JJ, Thompson BG, et al. Extracranial-intracranial bypass for stroke-is this the end of the line or a bump in the road? Neurosurgery. 2012;71(3):557-61.

11. Carlson AP, Yonas H, Chang YF, Nemoto EM. Failure of cerebral hemodynamic selection in general or of specific positron emission tomography methodology?: Carotid Occlusion Surgery Study (COSS). Stroke. 2011;42(12):3637-9.

12. Low SW, Teo K, Lwin S, Yeo LL, Paliwal PR, Ahmad A, et al. Improvement in cerebral hemodynamic parameters and outcomes after superficial temporal artery-middle cerebral artery bypass in patients with severe stenoocclusive disease of the intracranial internal carotid or middle cerebral arteries. $J$ Neurosurg. 2015;123(3):662-9.

13. Komotar RJ, Starke RM, Otten ML, Merkow MB, Garrett MC, Marshall RS, et al. The role of indirect extracranial-intracranial bypass in the treatment of symptomatic intracranial atheroocclusive disease. J Neurosurg. 2009;110(5):896-904.

14. Matano F, Murai Y, Tateyama K, Tamaki T, Mizunari T, Matsukawa H, et al. Long-term patency of superficial temporal artery to middle cerebral artery bypass for cerebral atherosclerotic disease: factors determining the bypass patent. Neurosurg Rev. 2016;39(4):655-61.

15. Teo K, Choy DK, Lwin S, Ning C, Yeo TT, Shen L, et al. Cerebral hyperperfusion syndrome after superficial temporal artery-middle cerebral artery bypass for severe intracranial steno-occlusive disease: a case control study. Neurosurgery. 2013;72(6):936-42; discussion 42-3.

16. Gonzalez NR, Dusick JR, Connolly M, Bounni F, Martin NA, Van de Wiele B, et al. Encephaloduroarteriosynangiosis for adult intracranial arterial steno-occlusive disease: long-term single-center experience with 107 operations. J Neurosurg. 2015;123(3):654-61.

17. Kawashima M, Noguchi T, Yakushiji Y, Takase Y, Matsushima T. Leptomeningeal collateral and cerebral hemodynamics in patients with ICA and MCA steno-occlusion. Neurol Res. 2011;33(3):30713. 
18. Singer OC, Berkefeld J, Nolte $\mathrm{CH}$, Bohner G, Reich A, Wiesmann M, et al. Collateral vessels in proximal middle cerebral artery occlusion: the ENDOSTROKE study. Radiology. 2015;274(3):851-8.

19. Arias EJ, Dunn GP, Washington CW, Derdeyn CP, Chicoine MR, Grubb RL, Jr., et al. Surgical Revascularization in North American Adults with Moyamoya Phenomenon: Long-Term Angiographic Follow-up. J Stroke Cerebrovasc Dis. 2015;24(7):1597-608.

20. Esposito G, Amin-Hanjani S, Regli L. Role of and Indications for Bypass Surgery After Carotid Occlusion Surgery Study (COSS)? Stroke. 2016;47(1):282-90.

21. Chou CW, Chang JH, Lin SZ, Cho DY, Cheng YW, Chen CC. Extracranial-intracranial (EC-IC) bypass of symptomatic middle cerebral artery (MCA) total occlusion for haemodynamic impairment patients. Br J Neurosurg. 2012;26(6):823-6.

22. Kim YS, Kim BJ, Noh KC, Lee KM, Heo SH, Choi HY, et al. Distal versus Proximal Middle Cerebral Artery Occlusion: Different Mechanisms. Cerebrovasc Dis. 2019:1-7.

23. Andrew NE, Kim J, Thrift AG, Kilkenny MF, Lannin NA, Anderson CS, et al. Prescription of antihypertensive medication at discharge influences survival following stroke. Neurology. 2018;90(9):e745-e53.

24. Tully PJ, Debette S, Dartigues JF, Helmer C, Artero S, Tzourio C. Antihypertensive Drug Use, Blood Pressure Variability, and Incident Stroke Risk in Older Adults: Three-City Cohort Study. Stroke. 2016;47(5):1194-200.

25. Tziomalos K, Giampatzis V, Bouziana SD, Spanou M, Kostaki S, Papadopoulou M, et al. Effect of antihypertensive treatment on the long-term outcome of patients discharged after acute ischemic stroke. Clin Exp Hypertens. 2017;39(3):246-50.

\section{Figures}




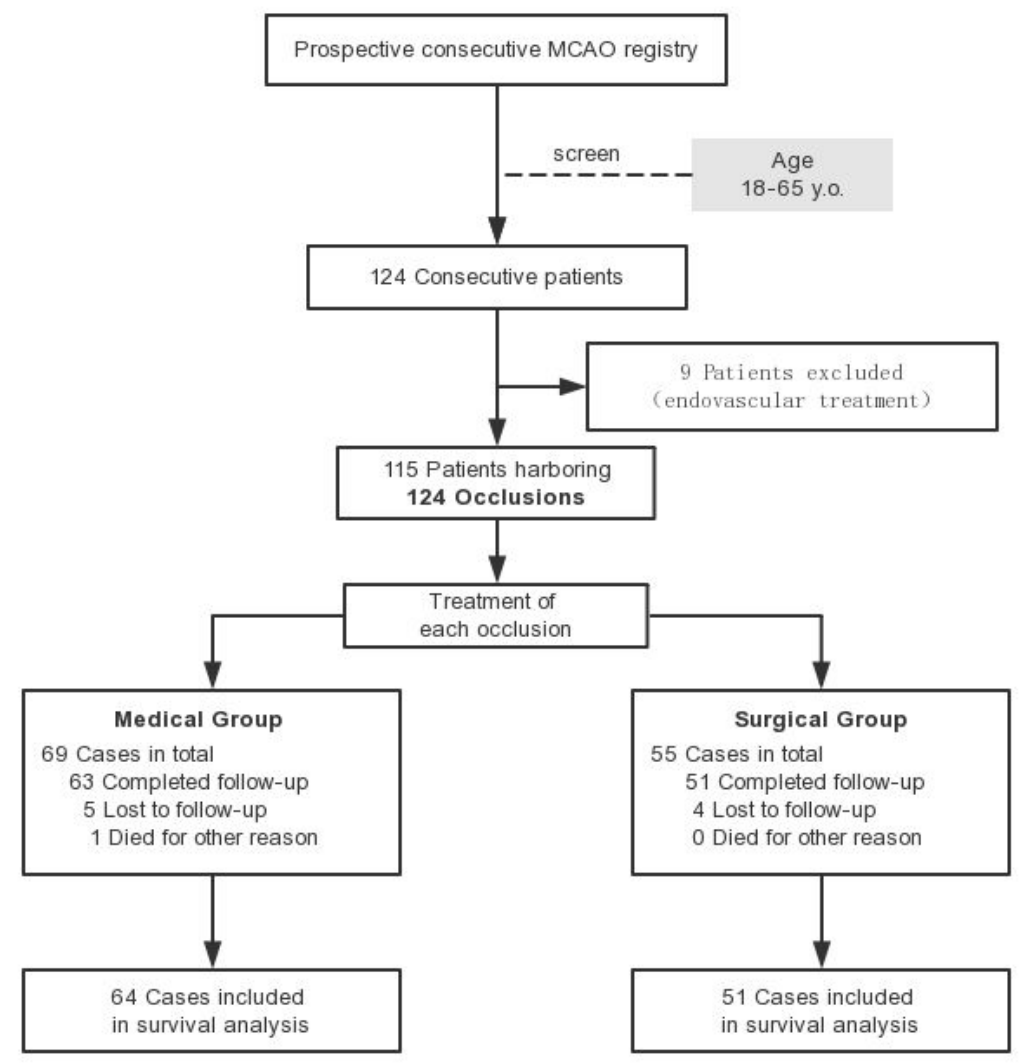

Figure 1

Schematic Illustration of the Study Protocol 


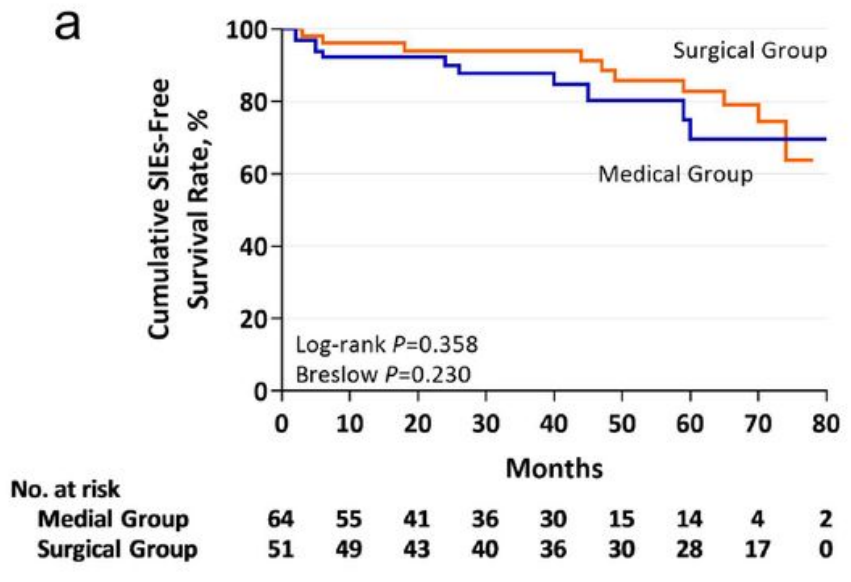

b

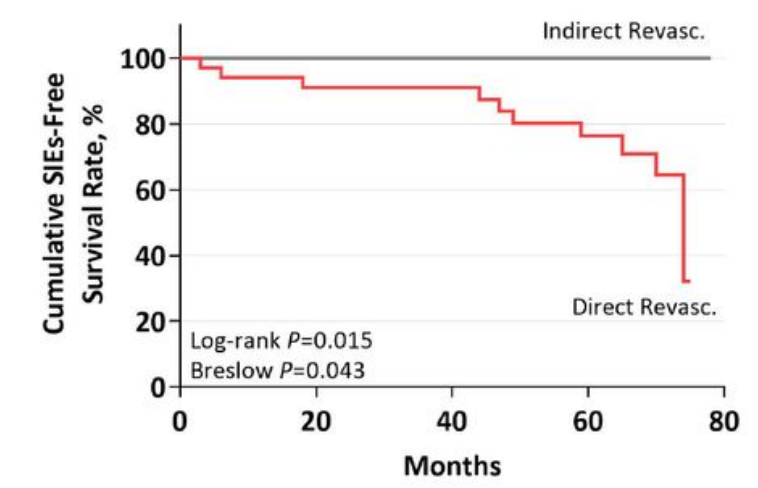

No. at risk

$\begin{array}{llllll}\text { Direct Revasc. } & 34 & 28 & 25 & 20 & 0 \\ \text { Indirect Revasc. } & 16 & 14 & 10 & 7 & 0\end{array}$

C

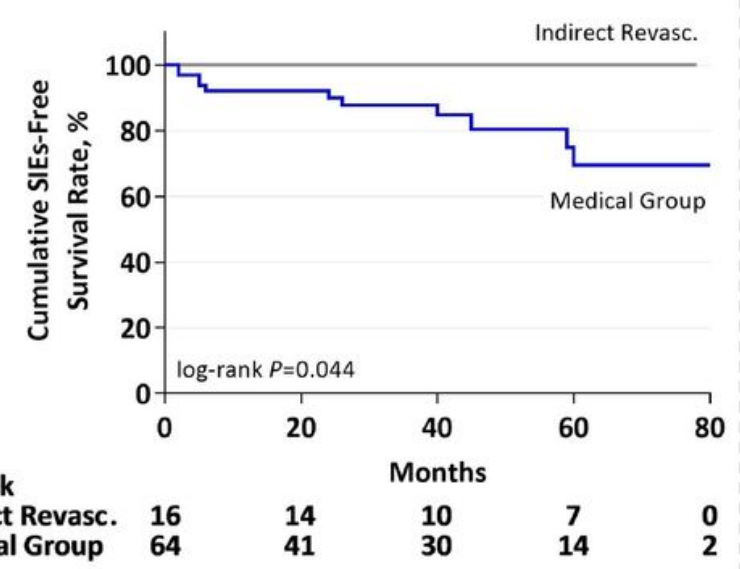

d
No. at risk

Direct Revasc.

Medical Group
20 Log-rank $P=0.972$

Months
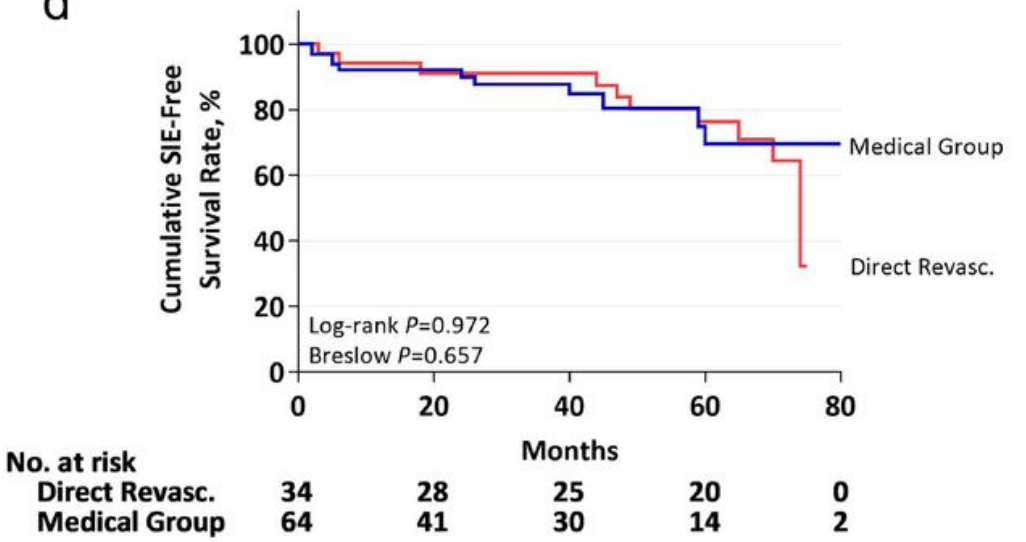

No. at risk Medical Group 64

\section{Figure 2}

Kaplan-Meier estimates of Different Therapies and Procedures (a) The survival rate showed no difference between surgical group and medical group. (b) Indirect revascularization subgroup showed a significantly higher survival rate than direct revascularization subgroup. (c) Indirect revascularization was superior to medical treatments for its higher SIE-free survival rate. (d) Survival rates were indifferent between medical treatments and direct revascularization. Abbreviation: Revasc. Revascularization 

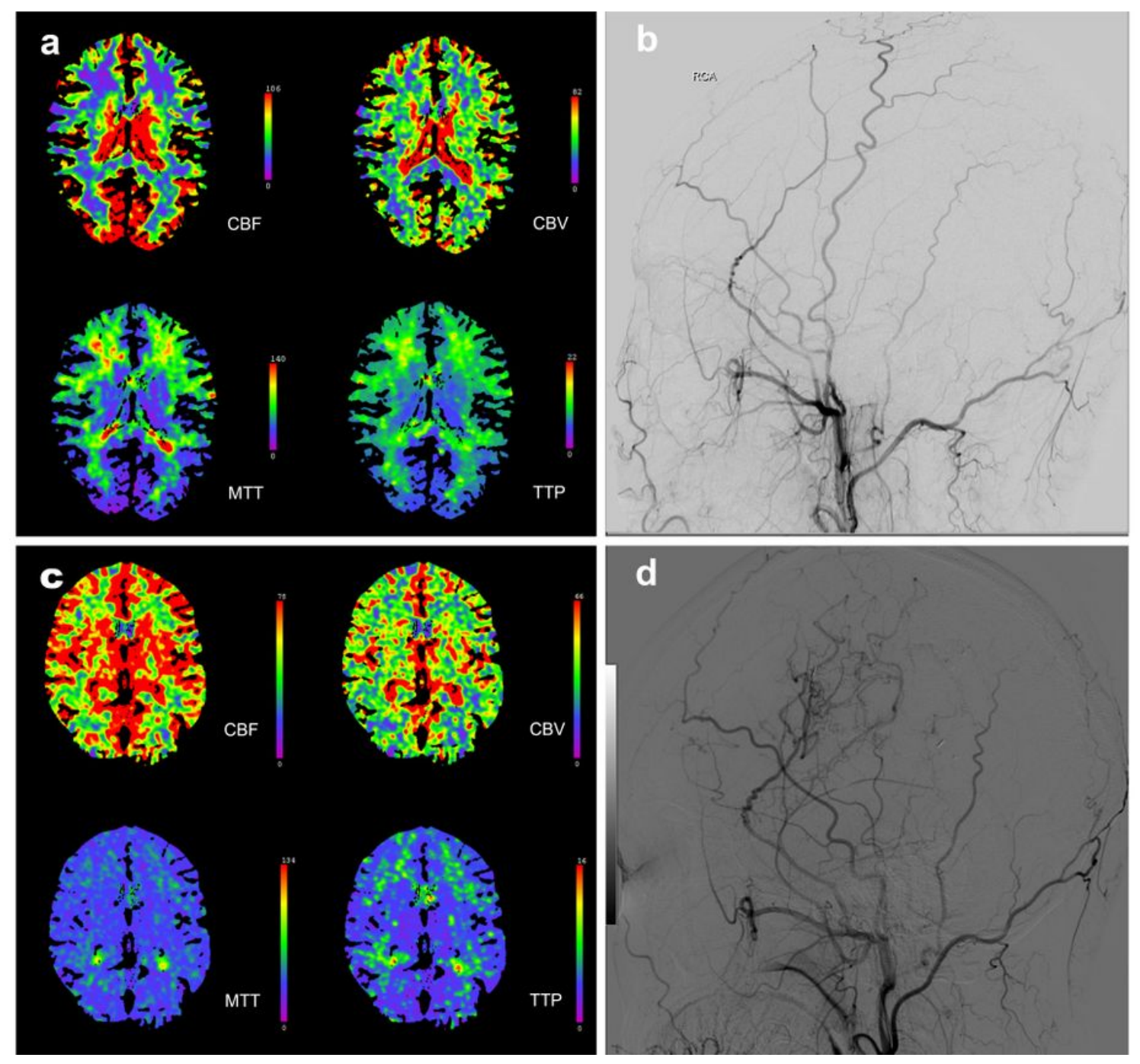

\section{Figure 3}

Improvement on Cerebral Perfusion after mEDAS A female MCAO patient aged 56y, with a chief complain about weakness on left extremities. The preoperative CTP (a) revealed significant prolongations of MTT and TTP, and reductions of CBF on bilateral frontoparietal lobes, which was worse on the right.

Extracranial compensatory collateral artery was not found in preoperative DSA (b). The patient received an inpatient follow-up in 7 months after mEDAS. CTP revealed a significant prolonged TTP on bilateral fontroparietal lobes but with recovered MTT and CBF (c). DSA revealed the presence of compensatory collateral arteries proliferating from STA to ischemic territories (d). Abbreviations: MCAO, middle cerebral arterial occlusion; CTP, computed tomographic perfusion; MTT, mean transit time; TTP, time to peak; CBF, cerebral blood flow; DSA, digital subtraction angiography; mEDAS, modified encephalo-duro-arteriosynangiosis; STA, superficial temporal artery. 